\title{
Diagnostics for magnetic confinement fusion research
}

\author{
Arthur Weller ${ }^{*}$ \\ Max-Planck-Institut für Plasmaphysik, IPP-EURATOM-Association, Boltzmannstr. 2 D-85748 Garching, Germany
}

\begin{abstract}
Significant progress towards the development of an attractive fusion energy source based on magnetic or inertial plasma confinement has been achieved within the international fusion energy program. High-level diagnostics capabilities are required to characterize fusion plasmas and to achieve a sound physics basis to design a fusion power plant. A large variety of different measuring techniques is used, most of them based on the detection of electromagnetic radiation in a wide range of wavelengths or of particles emitted from the plasma. Active probing by laser and particle beams permit to measure local plasma parameters directly, whereas passive measurements and imaging methods require unfolding and tomographic reconstruction techniques in order to obtain the spatial source distribution. Most diagnostics systems are limited in the accessible parameter range, in accuracy, temporal and spatial resolution, energy resolution and hardiness in a harsh environment, so that redundancy and complementarity of different methods is desirable. A considerable synergy exists between plasma diagnostics for fusion and astrophysics research. In particular, novel imaging detectors developed for the observation of astrophysical objects may be applied to fusion devices, too. An overview of diagnostics requirements, measuring techniques and selected results are presented with an emphasis of imaging diagnostics in toroidal magnetic fusion devices. (C) 2001 Elsevier Science. All rights reserved
\end{abstract}

Keywords: fusion; magnetic confinement; diagnostics; imaging; tomography

\section{Introduction}

A major part of the worldwide fusion research activities is devoted to magnetic plasma confinement devices. Until now, the fusion triple product, which is a figure of merit for magnetically confined plasmas, could be raised to values of $n T \tau_{E}>10^{21} \mathrm{~m}^{-3} \mathrm{keVs}$, which is within a factor of about 3 below the limit for ignition in a deuterium tritium plasma. Here, $n$ is the plasma density, $T$ is the ion temperature, and $\tau_{E}$ is the energy confinement time defined by $\tau_{E}=3 n \cdot T \cdot V / P_{h}$, with the total plasma energy ( $V$ is the plasma volume) divided by the heating power. Within the various magnetic confinement concepts the tokamak is the most advanced system. Actually, in the largest presently existing tokamak JET (Joint European Torus) [1] a fusion power of up to $16 \mathrm{MW}$ was already produced. The next step towards a fusion power reactor will be the ITER device [2], in which a fusion power of about $500 \mathrm{MW}$ and a fusion gain of $Q>10$ is anticipated, where $Q$ is the fusion power normalised to the heating power. This is already at a

\footnotetext{
* Corresponding author. Tel.: +49 (0)89 3299 2225; fax: +49 (0)89 3299 1812; e-mail: arthur.weller@ipp.mpg.de.
} 
level of a power plant, and hence fusion research will be closely interlinked with technological programs.

The stellarator concept is an alternative magnetic confinement system, which does not require current drive, and hence is principally qualified for steady state operation. Presently, the largest device is the Large Helical Device (LHD), in which reactor relevant values of the volume averaged plasma beta up to $\beta=5 \%$ could be achieved [3], however, in a low magnetic field configuration and hence in a low temperature regime. Here, beta is the normalized plasma pressure given by $\beta=n \cdot T /\left(B^{2} / 2 \mu_{0}\right)$. The next stellarator device will be Wendelstein W7-X, which is presently under construction [4]. A major goal is to demonstrate quasi-stationary plasma confinement in an optimized stellarator configuration with relevant plasma parameters.

In many experiments an extensive set of different diagnostics is used. In particular, a number of new developments have been made based on the progress of detector and component fabrication technologies, of data acquisition electronics and of analysis methods. This has greatly fostered the understanding of fusion physics and influenced the design of new devices. The scope of this invited paper is limited to a brief general overview of diagnostics techniques used in magnetic fusion devices highlighting a few selected diagnostics and results with an emphasis on two-dimensional (2D) plasma imaging. Comprehensive review papers on diagnostics can be found in a series of papers published in Fusion Science and Technology, Volume 53, Number 2, February 2008. A compilation dedicated to ITER can be found in [5], and aspects of diagnostics for steady state plasmas are reviewed in ref. [6].

The present paper is organized as follows: In Section 2 the diagnostics requirements related to the main physics issues are discussed, while in Section 3 some general diagnostics properties and selected diagnostic techniques are listed. In Section 4 examples of selected measurements and results are compiled with emphasis on plasma imaging diagnostics. Some concluding remarks are given in Section 5.

\section{Physics issues and diagnostics requirements}

Despite of the experimental and theoretical achievements of important milestones there are still open physics and engineering issues from which related diagnostic development needs can be deduced. One important area is the control of magnetohydrodynamic (MHD) instabilities. The avoidance or mitigation of current disruptions is a key issue for ITER and tokamak fusion energy devices. In particular, 2D and 3D data on the distribution of halo currents, on secondary runaway energy distributions and fast infrared camera measurements are highly desirable to resolve issues connected with forces and heat loads. Likewise, real time diagnostics that can identify the onset of disruptions in an early phase are important to deliver trigger for mitigation systems. Other MHD stability issues are associated with edge localized modes (ELM), which are beneficial for impurity control but can cause unacceptable heat loads in ITER. Here, significant efforts are made to increase the temporal and spatial resolution of profile and transient heat load measurements during ELMs as well as in inter-ELM phases and of transient heat load measurements [7],[8] in order to make reliable model predictions and to achieve profile control. Fast recognition and imaging of neoclassical tearing modes (NTM) is a prerequisite for NTM control by local current drive or heating. Reconstruction of 2D and 3D mode structures of Alfvén instabilities, ELMs, NTMs, pressure driven modes and resistive wall modes (RWM) are of great importance for comparing measurements with model calculations. Here, efforts concerning integrated data analysis of several diagnostics and, the use of advanced reconstruction algorithms and fast imaging techniques have to be continued.

Another important field requiring more detailed work is related to energy and particle transport. In particular, simultaneous measurements of density, temperature and electric field fluctuations including 2D characterization of turbulent flows are desirable to assess the role of turbulent transport. Measurements with high spatial resolution are needed in the region of transport barriers. A particular issue in stellarators and helical systems is the potential deterioration of flux surfaces by error fields. Visualization of 
the vacuum flux surfaces, therefore, is an important task.

Other important topical issues concern fast particle physics, current drive in connection with long pulse operation, and boundary physics together with plasma wall interaction. In particular, the last topic requires $3 \mathrm{D}$ measurements and imaging at the plasma edge. Most of the listed topics are interlinked, and therefore, integrated solutions of the various issues have to be found to establish self-sustained longpulse scenarios in tokamaks.

\section{Diagnostics techniques}

A large variety of different diagnostics is used in most magnetic confinement devices in order to determine plasma and magnetic configuration parameters. Besides measurements at the very edge of the plasma with material probes, non-invasive methods have to be applied to avoid mutual perturbations of measurements and plasma performance. A rough classification may be given according to basic properties of the different diagnostics systems:

(i) magnetic diagnostics make use of various flux loops by which the plasma current, the loop voltage, the plasma energy and plasma equilibrium quantities can be inferred. Particular components of magnetic perturbations are determined from pick-up coils distributed poloidally and toroidally around the torus. In stellarators, the geometry of the vacuum magnetic surfaces can be visualized by an electron beam injected on particular magnetic field lines.

(ii) passive photon detection is used by a number of measurements including bolometry, X-ray and visible bremsstrahlung measurements, spectroscopy and thermography. These passive measurements yield integrated intensities along diagnostic sightlines so that Abel inversion or tomographic reconstruction techniques [9], [10] have to be applied to deconvolve the measurements in order to get $1 \mathrm{D}$ or $2 \mathrm{D}$ images of the emission source. Since the limited number of measurements along different chords usually leads to an underdetermined problem for the reconstruction of the image, regularization algorithms such as the maximum entropy method have to be applied which provides an unbiased result given the data constraints and prior knowledge.
On the other hand, radiometry of electron cyclotron emission (ECE) gives the local electron temperature at the ECE resonance layer. 2D distributions of the electron temperature are obtained by ECE imaging (ECEI) onto linear antenna arrays. In this way the spatial resolution provided by the different sightlines is combined with the spatial resolution along each sightline in the major radius direction according to the change of the magnetic field and hence to the frequency range of the radiometers.

(iii) Likewise, passive particle detection methods are used to determine ion temperatures by charge exchange (CX) neutral particle analysis (NPA), fast ion losses and fluxes of fusion products. Tomographic imaging of neutron or gamma radiation on JET has provided important information on source distributions and confinement of fast ions from plasma heating and from fusion reactions [11], [12], [13].

(iv) Active photon scattering methods are based on launching electromagnetic probing beams into the plasma. In the case of interferometry, polarimetry and reflectometry microwave beams are used for probing

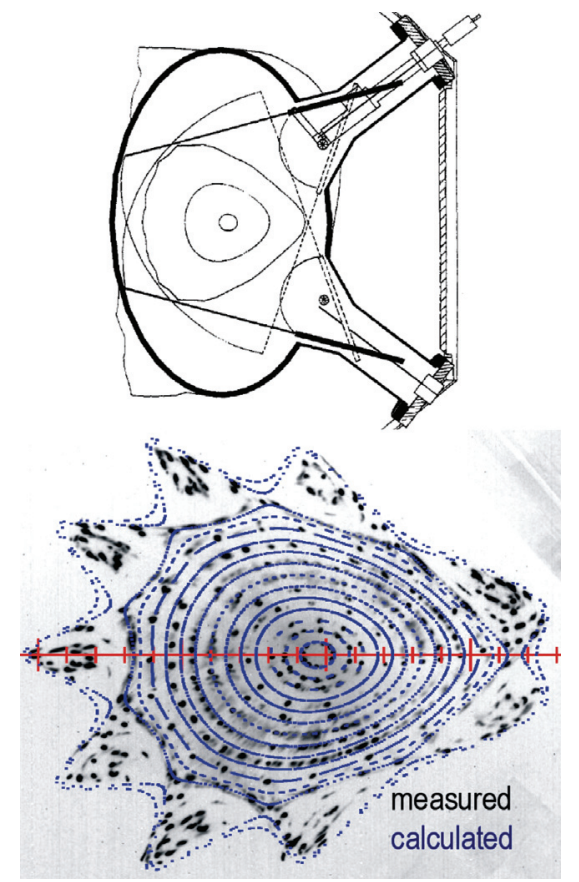

Fig. 1. Field line tracing measurements (with rotating scintillator rod system, top) in the Wendelstein W7-AS stellarator compared with calculations (thin blue dots). Points on each flux surface are connected by one field line - Courtesy M. Otte. 
the electron density or the poloidal magnetic field. Laser Thomson scattering and laser spectroscopy are widely used for the determination of the local electron temperature and density. Microwave imaging reflectometry (MIR) has been proposed for turbulence studies [14]. However, this is a very sophisticated method which requires further development.

(v) Correspondingly, active particle scattering diagnostics uses neutral beams or gas puffing, e.g. H-, $\mathrm{He}$ - or Li-beams. Important diagnostics based on detecting beam induced signals are CXrecombination spectroscopy (CXRS), active CX NPA and beam emission spectroscopy (BES). The latter is frequently used for imaging of MHD modes and density fluctuations [15]. Gas puff imaging at the plasma edge is another method for visualizing turbulent structures and flows [16]. Also, heavy ion beams of high energy (MeV range) are used to probe the radial electric field as well as density fluctuations in the plasma [17].

The different diagnostic measurements cover a wide spectral range. Imaging involves multi-scale structures ranging from the full plasma size down to objects of the order of gyro radius scales. Limitations of the spatial, temporal and spectral resolution, as well as in the accessibility range require complementary and redundant measurements and thus a large number of diagnostics systems.

The progress of fusion research leads to increased challenges for plasma diagnostics due to the harsh
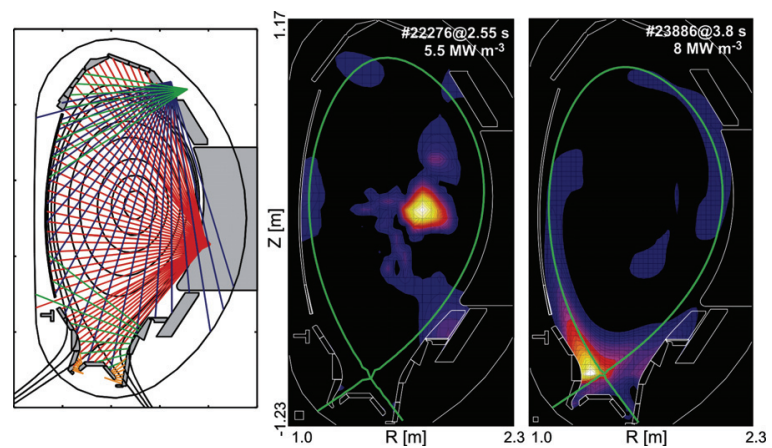

Fig. 2. Geometry of bolometer sightlines of bolometer cameras on ASDEX Upgrade (left). Tomographic reconstructions of total radiation for cases of central tungsten accumulation (middle) and of impurity control by central heating and nitrogen puffing - Courtesy J.C. Fuchs. environment in fusion relevant plasmas. In particular, sensitive diagnostics components close to the plasma such as optical mirrors and windows will suffer from high heat loads and coatings from sputtered material. Also, neutron induced changes of material properties including solid state detector damage are a serious problem for various diagnostics.

\section{Selected imaging diagnostics and results}

The first example refers to the electron beam mapping technique, which is used in stellarators to visualize the vacuum flux surfaces [18]. The measurement requires a small electron gun mounted on a manipulator. The electron beam is injected on various flux surfaces in another poloidal plane by scintillator rods sweeping across the surfaces or by a fixed scintillator mesh. The light spots produced by intersections of the beam and the scintillator are recorded by a video camera. Fig. 1 shows a sketch of the scintillator arrangement on Wendelstein W7-AS together with the resulting flux surface map which is composed of several camera exposures. Overlaid are dots resulting from field line tracing calculations which are in close agreement with the measurements.

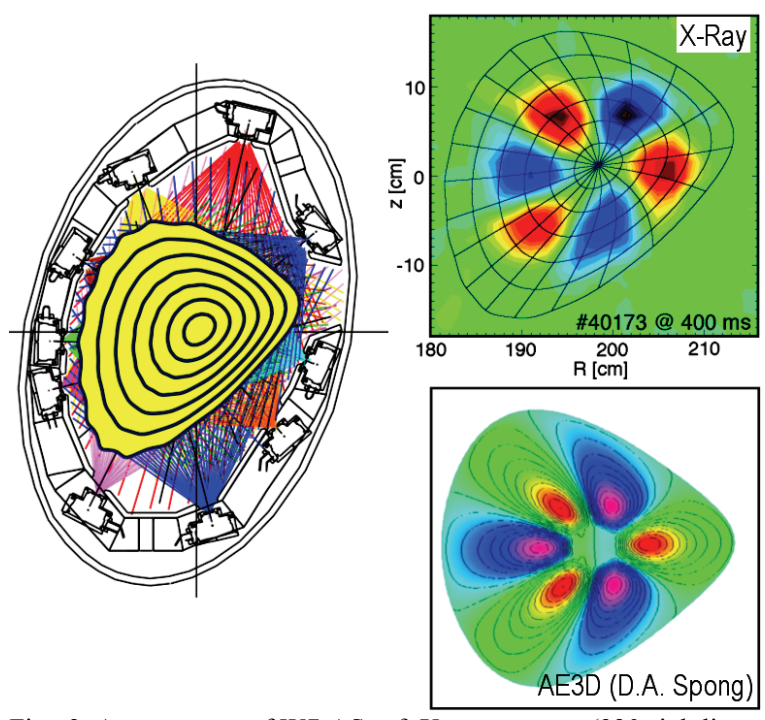

Fig. 3. Arrangement of W7-AS soft X-ray cameras (320 sightlines, left). Tomographic reconstruction of fluctuating emissivity (upper right) compared with prediction by AE3D MHD code (below). 


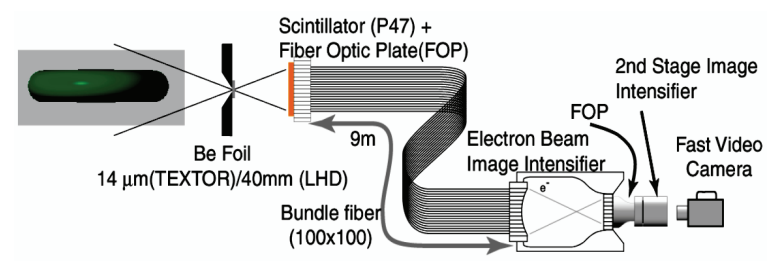

Fig. 4. Layout of the tangential X-ray imaging system used in LHD and TEXTOR - Courtesy S. Ohdachi.

Imaging of the total radiation by bolometer arrays is important for local energy balance analysis. Here, it is desirable to minimize radiation losses from the core plasma, but to allow for a radiating mantle at the plasma edge to avoid excessive heat loads on the divertor target plates. The sketch in fig. 2 shows the sightlines of several bolometer arrays in the ASDEX Upgrade tokamak (left) and the emissivity distributions for two experimental cases (middle and right). The bolometers used in ASDEX Upgrade are miniaturized low noise metal resistor bolometers [19] which are excited by a $50 \mathrm{kHz}$ sine wave and effectively suppress thermal drift and electromagnetic interferences. The tomographic reconstruction is based on a diffusion algorithm with strongly different diffusion coefficients along and perpendicular to the magnetic field [20]. The solution corresponds to minimized sources and sinks, given the constraints of the measured line integrals.

The spatial structure of MHD modes has been investigated by X-ray tomography in many experimen-
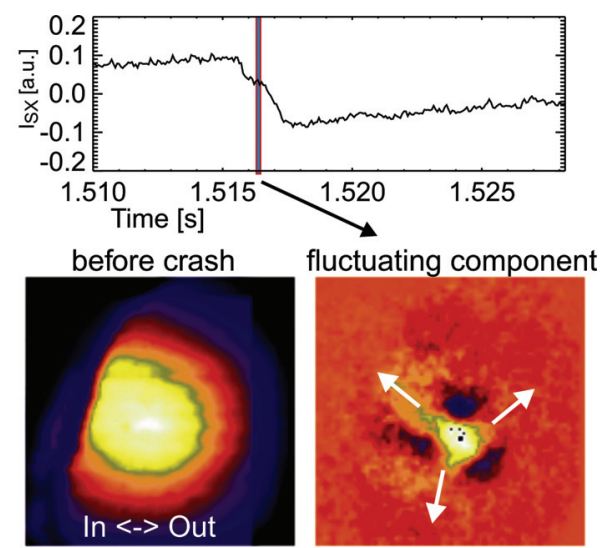

Fig. 5. Tangential X-ray images during a sawtooth instability in LHD. Top: time evolution of the central X-ray intensity. Bottom: raw and SVD filtered images during the collapse - Courtesy S. Ohdachi. tal devices. Mostly, energy integrating silicon diode arrays are used where the spectral range is given by a low-energy absorber foil and the high-energy transmission properties of the detectors. Fig. 3 (left part) shows the X-ray camera system used on Wendelstein W7-AS [21] consisting of 10 compact pinhole cameras arranged inside the vacuum vessel around the plasma (320 sightlines in total). The tomographic analysis for a particular case yields an extended $m=3$ mode structure after filtering the fluctuating part of the emissivity by a singular value decomposition (SVD) technique [22]. By comparison with the MHD code AE3D (D.A. Spong), the mode could be identified as a global Alfvén eigenmode (GAE) [23].

Whereas most X-ray measurements are made in a poloidal plane, approximate 2D emissivity distributions can be obtained by tangential views even without deconvolution since the emissivity does not vary so much along the sightlines. Thus, also higher poloidal mode numbers can be resolved. A tangential pinhole camera arrangement is used on LHD, and similarly on the TEXTOR [24], [25] and NSTX [26] tokamaks, where the X-ray emission is imaged onto a scintillator plate, which is coupled via fiber optics and an image intensifier stage to a fast video or framing camera. A sketch of the LHD system is shown in fig. 4. An example of raw images obtained during a $(m, n)=(3,2)$ sawtooth instability is shown in fig. 5 . The fluctuating $m=3$ component could be separated by SVD analysis of a time series of images.

Advances of microwave imaging techniques [27], [28] have provided new insights of the physics of MHD instabilities and plasma turbulence [29]. Fig. 6 contains a schematic diagram of an ECEI system as

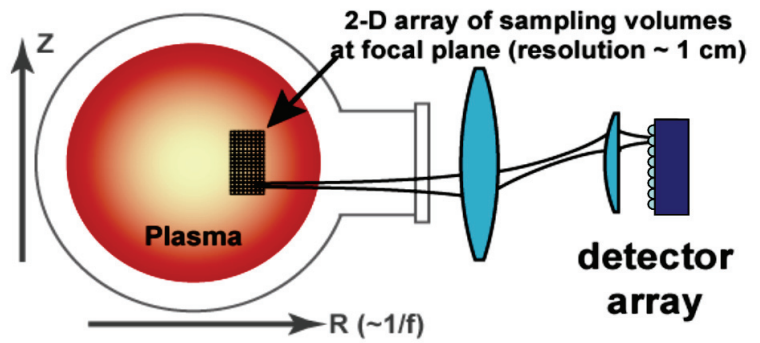

Fig. 6. Schematic diagram of an ECEI system. The sampling volume in the plasma can be adjusted by the imaging optics and changes of the toroidal magnetic field or the radiometer frequency - adapted from [27]. 


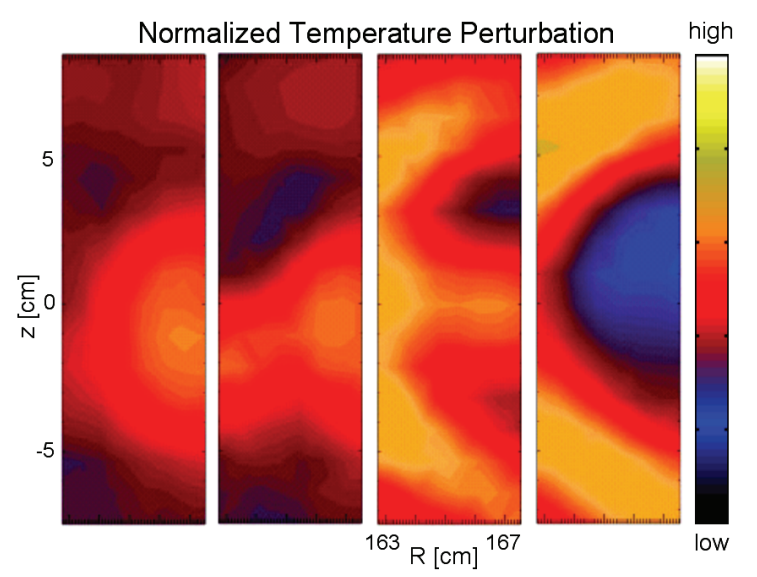

Fig. 7. 2D images of a sawtooth crash in TEXTOR. The ECEI sampling window covers an area around the sawtooth inversion radius at the high field side. The plasma centre is at the right edge of each frame in the horizontal midplane. The series shows that energy is expelled from the hot core in horizontally outward direction and a poloidal flow pattern appears. After reconnection the core is left at low temperature surrounded by a hotter region (right part). - Courtesy T. Munsat, H.K. Park, J. Boom, I.G.J. Classen.

used in TEXTOR. The 2D images shown in fig. 7 reveal details of the reconnection process during a sawtooth instability in TEXTOR [30]. The sampling volume was adjusted to cover a small area around the sawtooth inversion radius.

\section{Concluding remarks}

The present physics database used to design and build next step devices such as ITER relies to a large extent on diagnostics and computational capabilities. The realization of the ITER project will be a major step towards the development of fusion energy based on magnetic confinement. This has to be accompanied by diagnostics research and development in order to cope with operational constraints under the harsh conditions of burning long pulse plasmas. Also, the requirements regarding reliability will be much larger due to accessibility constraints.

The progress in solid state detector fabrication techniques for imaging purposes driven by astronomy, astrophysics missions and high energy particle physics may lead to synergy effects in fusion research. However, this may be limited to present fusion experiments with tolerable neutron fluxes. Imag- ing diagnostics based on microwaves and fusion products will be of increasing importance.

Other important topics are connected with stable long pulse plasma operation requiring active control on the basis of reliable real time diagnostic measurements. Some of the issues connected with the tokamak plasma current may be solved by stellarator devices.

\section{Acknowledgments}

The author is grateful to G.D. Conway and H.J. Hartfuss for stimulating discussions and information on microwave diagnostics and turbulence studies. The provision of information and material by J.C. Fuchs, M. Otte, S. Ohdachi, J. Boom, I.G.J. Classen, T. Munsat, H.K. Park, B. Huber, T. Lunt, M.A. Van Zeeeland and $\mathrm{H}$. Thomsen is gratefully acknowledged.

\section{References}

[1] M. Keilhacker, et al., Nucl. Fusion 39 (2) (1999) 209-234.

[2] Y. Shimomura, Journal of Nuclear Materials 329-333 (Part 1) (2004) 5-11

[3] A. Komori, et al., Nucl. Fusion 49 (10) (2009) 104015.

[4] L. Wegener, Fusion Engineering and Design 84 (2-6) (2009) 106-112.

[5] A. J. H. Donné, et al., Nucl. Fusion 47 (6) (2007) S337.

[6] H. J. Hartfuss, et al., Plasma Phys. Control. Fusion 48 (2006) R83-R150.

[7] F. Romanelli, et al., Nucl. Fusion 49 (10) (2009) 104006.

[8] T. Eich, et al., Journal of Nuclear Materials 337-339 (2005) 669-676.

[9] L. C. Ingesson, et al., Fusion Science and Technology 53 (2) (2008) 528-576.

[10] R. S. Granetz, et al., Nucl. Fusion 28 (3) (1988) 457-476.

[11] F. B. Marcus, et al., Plasma Phys. Control. Fusion 33 (4) (1991) 277-287.

[12] M. Sasao, et al., Fusion Science and Technology 53 (2) (2008) 604-639.

[13] V. G. Kiptily, et al., Nucl. Fusion 49 (6) (2009) 065030.

[14] E. Mazzucato, et al., Phys. Plasmas 9 (5) (2002) 1955-1961.

[15] G. R. McKee, et al., Rev. Sci. Instrum. 74 (3) (2003) 20142019.

[16] S. J. Zweben, et al., Phys. Plasmas 9 (5) (2002) 1981-1989.

[17] P. M. Schoch, et al., Rev. Sci. Instrum. 59 (8) (1988) 16461651.

[18] R. Jaenicke, et al., Nuclear Fusion 33 (5) (1993) 687-704.

[19] K. F. Mast, et al., Rev. Sci. Instrum. 62 (3) (1991) 744-750. 
[20] J. C. Fuchs, et al., Europhysics Conference Abstracts (Proc. 21th EPS Conf. on Contr. Fusion and Plasma Phys.) Montpellier, France, Vol. 18B (1994) 1308-1311.

[21] C. Görner, et al., Europhysics Conference Abstracts (Proc. 24th EPS Conf. on Contr. Fus. \& Plas. Phys.) Berchtesgaden, Vol. 21A (1997) 1625

[22] A. Weller, et al., Physics of Plasmas 8 (3) (2001) 931-956.

[23] D. A. Spong, personal communication (2008).

[24] S. Ohdachi, et al., Rev. Sci. Instrum. 74 (3) (2003) 21362143.

[25] S. Ohdachi, personal communication (2009).

[26] C. E. Bush, et al., Rev. Sci. Instrum. 79 (10) (2008) 10 E928.

[27] H. Park, et al., Rev. Sci. Instrum. 75 (10) (2004) 3787-3792.

[28] B. Tobias, et al., Rev. Sci. Instrum. 80 (9) (2009) 093502.

[29] G. D. Conway, Plasma Phys. Control. Fusion 50 (12) (2008) 124026.

[30] T. Munsat, et al., Nucl. Fusion 47 (11) (2007) L31. 